\title{
Excesso de peso e consumo alimentar em adolescentes de escolas públicas no norte de Minas Gerais, Brasil
}

\author{
Excess weight and food consumption of adolescents \\ in public schools in northern Minas Gerais state, Brazil
}

Lucinéia de Pinho ${ }^{1}$

Eliete Fernandes Flávio ${ }^{2}$

Sérgio Henrique Sousa Santos ${ }^{1}$

Ana Cristina de Carvalho Botelho ${ }^{1}$

Antônio Prates Caldeira ${ }^{1}$

${ }^{1}$ Programa de PósGraduação em Ciências da Saúde, Universidade Estadual de Montes Claros. Campus Universitário Professor Darcy Ribeiro, Vila Mauriceia. 39.401-089 Montes Claros MG Brasil. lucineiapinho@hotmail.com ${ }^{2}$ Hospital Universitário, Universidade Estadual de Montes Claros.
Abstract This study sought to identify the prevalence of excess weight and food consumption patterns among adolescents attending public schools in northern Minas Gerais state. A cross-sectional study was conducted with a random and representative sample of adolescents. Demographic and economic data were collected. Food consumption was evaluated by applying a semi-quantitative food questionnaire. Body weight and height were measured to assess the nutritional status based on the Body Mass Index (BMI) by Age. The sexes were compared by Student's t test (comparison of samples) and chi-square test (goodness of fit) at a level of significance of $p<0.05 .535$ adolescents were evaluated and prevalence of excess weight was detected in $18.5 \%$ of the group. BMI $(p=0,001)$ was statistically different between the sexes and higher for girls. The average energy consumption of the group was $3096.30 \mathrm{kcal} / \mathrm{day}$. Fiber consumption was insufficient in $35 \%$ of the adolescents, vitamin $A$ and calcium in $80 \%$ and unsaturated fats in 100\%. Carbohydrate and cholesterol were consumed in excess by $20.6 \%$ and $48 \%$, respectively. The prevalence of excess weight and inadequate food consumption is of great concern for the population studied and requires appropriate nutritional intervention.

Key words Adolescents, Food consumption, $\mathrm{Nu}$ tritional status
Resumo Este estudo objetivou identificar a prevalência excesso de peso e o consumo alimentar entre adolescentes de rede pública de ensino ao norte de Minas Gerais. Desenvolveu-se estudo transversal com amostra aleatória e representativa de adolescentes. Foram coletadas informações demográficas e econômicas. O consumo alimentar foi avaliado pela aplicação de questionário semiquantitativo. Aferiu-se peso e altura para avaliação do estado nutricional com base no Índice de Massa Corporal (IMC) por Idade. A comparação entre os gêneros foi conduzida por meio do teste $t$ de Student (médias) ou qui-quadrado (proporções), assumindo-se um nível de significância de $p<0,05$. Foram avaliados 535 adolescentes e registrada uma prevalência de excesso de peso para 18,5\%. Houve diferença significativa entre os gêneros para o IMC ( $p=0,001$ ), maior entre as meninas. O consumo médio de energia foi de 3.096,30kcal/dia. Observou-se consumo insuficiente de fibras em $35 \%$ dos adolescentes, de cálcio e vitamina A em $80 \%$ e gorduras insaturadas em 100\%. Carboidratos e colesterol eram consumidos em excesso por $20,6 \%$ e $48 \%$, respectivamente. A prevalência do excesso de peso e o consumo alimentar inadequado mostrouse preocupante para a população estudada e demandam adequada intervenção nutricional.

Palavras-chave Adolescentes, Consumo alimentar, Estado nutricional 


\section{Introdução}

Nos últimos anos, a incidência de obesidade entre crianças e adolescentes cresceu consideravelmente em várias partes do mundo ${ }^{1}$. Nos Estados Unidos a obesidade nessa faixa etária é considerada um importante problema de saúde públi$\mathrm{Ca}^{2}$, e o fato da situação não ser diferente no Brasil ressalta a importância do tema para profissionais e gestores da área da saúde ${ }^{3}$. O desenvolvimento da obesidade infanto-juvenil se associa a uma complexa rede de variáveis, dentre as quais se destacam a falta de atividade física e hábitos alimentares inadequados ${ }^{4}$.

As particularidades da adolescência, que se caracteriza como um período de rápidas transformações corporais e alterações psicossociais, influenciam notadamente os hábitos e as recomendações alimentares, o que torna a questão ainda mais desafiadora. A dieta representa, nesta fase, um dos principais componentes do estilo de vida a favorecer o desenvolvimento da obesidade e suas comorbidades. Por isso, deve ser especialmente monitorada.

Atualmente é comum que adolescentes substituam as principais refeições por lanches hipercalóricos ou não façam algumas refeições importantes como o café da manhã. Além disso, eles consomem muitos alimentos ricos em açúcar, carboidratos refinados e gordura saturada e poucas frutas e hortaliças, adotando inclusive dietas monótonas ou modismos alimentares ${ }^{5}$. $\mathrm{O}$ fenômeno de mudanças de hábitos alimentares, inserido no contexto das transições demográficas e epidemiológicas presenciadas em todo o mundo, é denominado transição nutricional e envolve não apenas alterações nas fontes alimentares e desequilíbrio energético como também das doenças relacionadas à dieta. Nos países em desevolvimento, um exemplo claro de transição nutricional é a associação positiva entre a renda da população e a prevalência da obesidade e suas comorbidades entre crianças e adultos jovens. Além do aumento no poder aquisitivo, nas últimas décadas houve aumento na disponibilidade de alimentos semiprontos e de fácil preparo, o que estimula o consumo desbalanceado de nutrientes ${ }^{6}$.

Estudos sobre o padrão alimentar de adolescentes são fundamentais para reforçar os hábitos saudáveis e atenuar aqueles indesejáveis ou mesmo maléficos. Nesse sentido é importante identificar o consumo de macronutrientes e também de micronutrientes que, quando inadequado, pode trazer graves danos à saúde ${ }^{7}$.
Deve-se destacar ainda que a elaboração de um plano dietético para um determinado grupo requer uma avaliação prévia de suas condições físicas e hábitos alimentares, os quais sofrem influência cultural e regional. Nesse contexto, a fim de subsidiar planos de ação de combate ao sobrepeso e obesidade em adolescentes da rede pública de ensino ao Norte de Minas Gerais, este trabalho teve o objetivo de investigar o consumo alimentar e a prevalência de excesso de peso dessa população.

\section{Metodologia}

Trata-se de um estudo descritivo de corte transversal, realizado com adolescentes de ambos os gêneros, matriculados na rede pública de ensino da cidade de Montes Claros (MG). Localizada ao norte do estado, a cidade representa o principal polo urbano da região e tem aproximadamente 360 mil habitantes.

Os participantes foram selecionados por amostragem aleatória por conglomerados. O tamanho amostral de 474 indivíduos foi calculado com o software Epi Info versão 3.5.2 e baseado no número total de alunos de ensino fundamental matriculados no $6^{\circ}$ ao $9^{\circ}$ ano de escolas da rede pública da zona urbana do município (a zona rural foi excluída por questões logísticas e por atender menos de 3\% dos estudantes da rede pública local).

Foram considerados os seguintes critérios para o cálculo amostral: a) número total de alunos; b) nível de confiança de 95\%; c) erro amostral de 5\%; d) fator de correção para o desenho amostral ("deff”) igual a 2; e e) prevalência de obesidade de $20 \%$, considerando dados de estudos com adolescentes brasileiros ${ }^{8}$.

A amostra foi escolhida aleatoriamente de acordo com a técnica de amostragem por conglomerados em dois estágios. No primeiro estágio foi realizada a seleção das escolas, por meio de método de seleção com probabilidades proporcionais ao tamanho. No segundo foi realizada a seleção das turmas por amostragem aleatória simples, entrevistando-se todos os alunos das turmas selecionadas.

Foram coletadas informações sociodemográficas (gênero, idade, série, turno da aula, nível socioeconômico e escolaridade dos pais) dos participantes. A idade, considerada por faixa etária, e o nível socioeconômico foi declarado pelos pais em um formulário e classificado, de acordo com a renda familiar per capita (em salários mínimos 
obtidos pela família no mês anterior à entrevista) em $<1$ salário mínimo ou $\geq 1$ salário mínimo.

As medidas antropométricas de peso e estatura foram aferidas na escola. Para a aferição do peso corporal, foi solicitado que os adolescentes vestissem roupas leves e permanecessem descalços, sendo então posicionados sobre uma balança eletrônica portátil Marte ${ }^{\circledR}$ classe III, modelo LC200-PS, com capacidade para 199,95 kg, mínima de $1 \mathrm{~kg}$ e precisão de $50 \mathrm{~g}$.

Para a medida da altura, foi utilizado um estadiômetro vertical Altura exata ${ }^{\circledR}$ com escala numérica bilateral de 35 a $213 \mathrm{~cm}$ e resolução de 0,1 $\mathrm{cm}$. Nessa medição, os jovens mantinham-se descalços, com os pés juntos, calcanhares encostados na parede, em postura ereta, com olhar fixo no horizonte, sem flexão ou extensão da cabeça. Em seguida, posicionava-se a barra horizontal do estadiômetro até mantê-la apoiada sobre a cabeça do adolescente, sendo então efetuada a leitura da altura em centímetros.

As medidas de peso e altura foram realizadas em duplicata, e o valor médio obtido para cada participante foi usado para o cálculo do índice de massa corporal (IMC) para idade, em escores-z, para avaliação do estado nutricional. Adotou-se como referência a proposta da Organização Mundial da Saúde para crianças e adolescentes de 5 a 19 anos $^{9}$.

A avaliação do consumo habitual de alimentos foi realizada por meio da aplicação do Questionário de Frequência Alimentar para Adolescentes (QFAA). Trata-se de um instrumento semiquantitativo que oferece sete opções de consumo de 94 alimentos: nunca; menos de uma vez ao mês; de 1 a 3 vezes no mês; 1 vez por semana; de 2 a 4 vezes por semana; 1 vez ao dia; 2 ou mais vezes ao $\mathrm{dia}^{10}$. O questionário foi respondido pelos próprios adolescentes na escola.

Os dados do QFAA foram organizados em planilha para análise do valor nutricional das dietas individuais. As frequências de consumo dos diferentes itens alimentares foram transformadas para valores diários. Utilizando-se o software Diet Pró ${ }^{\circledR}$, os cálculos nutricionais foram realizados para todos os alimentos ingeridos.

Para a classificação da adequação energética e do consumo dos nutrientes, utilizaram-se pontos de corte baseados na referência Dietary Reference Intakes (DRI $)^{11}$. A adequação de energia foi calculada com base na ingestão energética de cada adolescente e sua necessidade, segundo a referência Estimated Energy Requirement (EER) e o percentual de macronutrientes foi considerado em relação ao Valor Energético Total (VET) e avaliado segundo a referência Acceptable Macronutrients Distribuition Range (AMDR). Para ferro, vitamina $\mathrm{C}$, vitamina A e zinco calculou-se a prevalência de inadequação, utilizando o valor da referência Estimated Average Requirement (EAR) como ponto de corte. Para cálcio, ûbras e sódio, utilizou-se o valor da referência Adequate Intake (AI).

O teor e a qualidade das gorduras foram avaliados de acordo com as indicações do Comitê de Nutrição da Academia Americana de Pediatria $^{12}$, que recomenda o consumo de ácidos graxos saturados $\leq 10 \%$ das gorduras totais, de ácidos graxos poliinsaturados $\geq 10 \%$, e de coleste$\mathrm{rol} \leq 300 \mathrm{mg} / \mathrm{dia}$.

Antes de se efetivar a pesquisa, um estudo piloto com 26 adolescentes de ambos os gêneros foi realizado a fim de aperfeiçoar os instrumentos e os procedimentos metodológicos. Completada essa fase, a coleta de dados, realizada durante o segundo semestre de 2011, foi conduzida em espaço reservado em cada escola, durante a jornada de aula, por equipe de pesquisadores treinados e calibrados.

Para manter a qualidade dos dados, foram excluídas da pesquisa as situações extremas, ou seja, as informações referentes aos indivíduos cuja ingestão energética apresentava-se inferior a 500 calorias (ou 5 itens do QFAA) ou superior a 7.000 calorias (ou 51 itens do QFAA).

O tratamento estatístico das informações foi realizado com o programa computacional Statistical Package for the Social Science (SPSS), versão 11.0. Para caracterização da amostra, utilizou-se estatística descritiva (média e desvio padrão). Para determinar a diferença entre os gêneros, utilizou-se teste $t$ de Student para comparação entre médias ou teste de qui-quadrado para comparar proporções, admitindo-se um nível de significância de 5\% $(p<0,05)$.

$\mathrm{O}$ estudo foi conduzido dentro dos preceitos éticos. Inicialmente, os adolescentes foram informados sobre o estudo. Aqueles que concordaram em participar receberam um "termo de consentimento" para ser preenchido pelos pais ou responsáveis, no qual constava uma breve explicação dos objetivos da pesquisa e metodologia assim como a afirmação de que não haveria identificação dos participantes. O estudo foi aprovado pelo Comitê de Ética em Pesquisa da Universidade Estadual de Montes Claros. 


\section{Resultados}

Participaram do estudo 535 adolescentes distribuídos em 25 turmas. O número de adolescentes que se recusaram a participar do estudo foi desprezível. Para análise dos dados, foram excluídos os questionários incompletos e aqueles referentes a consumos energéticos extremos. Os participantes do estudo tinham entre 11 e 17 anos de idade, sendo $68,0 \%(\mathrm{n}=364)$ do gênero feminino e $32,0 \%(n=171)$ do gênero masculino. Aproximadamente $80 \%$ dos adolescentes frequenta-

Tabela 1. Caracterização dos adolescentes de escolas públicas municipais em Montes Claros, MG, 2011.

\begin{tabular}{|c|c|c|}
\hline Variáveis & $\mathbf{N}$ & $\%$ \\
\hline \multicolumn{3}{|l|}{ Gênero } \\
\hline Masculino & 171 & 32,0 \\
\hline Feminino & 364 & 68,0 \\
\hline \multicolumn{3}{|l|}{ Idade (anos) } \\
\hline $11-12$ & 130 & 24,3 \\
\hline $13-14$ & 307 & 57,4 \\
\hline$>15$ & 98 & 18,3 \\
\hline \multicolumn{3}{|l|}{ Série } \\
\hline $6^{\circ}$ ano & 187 & 35,0 \\
\hline $7^{\circ}$ ano & 115 & 21,5 \\
\hline $8^{\circ}$ ano & 138 & 25,8 \\
\hline $9^{\circ}$ ano & 95 & 17,8 \\
\hline \multicolumn{3}{|l|}{ Turno } \\
\hline Manhã & 480 & 89,7 \\
\hline Tarde & 55 & 10,3 \\
\hline \multicolumn{3}{|c|}{ Escolaridade pai/responsável (anos) } \\
\hline$>8$ & 203 & 37,9 \\
\hline $5-7$ & 220 & 41,1 \\
\hline $0-4$ & 112 & 21,0 \\
\hline \multicolumn{3}{|l|}{ Renda familiar em SM${ }^{*}$} \\
\hline$<1 \mathrm{SM}$ & 341 & 63,7 \\
\hline$>1 \mathrm{SM}$ & 194 & 36,3 \\
\hline
\end{tabular}

vam o turno matutino de aula. Quanto à situação socioeconômica, observou-se que apenas $36,3 \%(\mathrm{n}=194)$ das famílias possuíam renda superior a um salário mínimo. A maioria dos pais tinha menos que sete anos de escolaridade (Tabela 1).

A idade, o peso e a estatura foram semelhantes entre os gêneros (Tabela 2). No entanto, o IMC foi menor em meninos que em meninas (Tabela 2).

Em relação ao perfil antropométrico dos adolescentes, observou-se que os meninos apresentaram maior proporção de baixo peso $(\mathrm{p}=0,001)$. A prevalência de sobrepeso $(\mathrm{p}=0,826)$ e obesidade $(\mathrm{p}=0,276)$ foram similares entre os gêneros.

O consumo energético e dos diferentes tipos de alimento pelos adolescentes é apresentado na Tabela 4. Foi observada diferença de consumo entre os gêneros apenas para cálcio.

A maioria dos adolescentes mostrou ter consumo adequado de nutrientes (Tabela 5). Destaca-se, porém, que $19,2 \%$ das meninas e $23,4 \%$ dos meninos apresentaram consumo superior ao recomendado para o grupo dos carboidratos ( $>65 \%$ do valor energético total). Foi observado também insuficiência no consumo de proteínas em $11,5 \%$ das meninas e $18,7 \%$ dos meninos, e de lipídios em $29,7 \%$ das meninas e $32,2 \%$ dos meninos. Em relação à qualidade dos lipídeos, identificou-se que a $100 \%$ dos participantes utilizavam ácidos graxos poliinsaturados em quantidades insuficientes e que o colesterol era consumido acima das quantidades recomendadas por $48 \%$ dos adolescentes.

Registrou-se o consumo insuficiente de micronutrientes como cálcio e vitamina A (Tabela 5). Também estava abaixo do adequado o consumo de fibras em $34,4 \%$ dos adolescentes do gênero feminino e 36,3\% do gênero masculino. O sódio era consumido acima dos valores recomendados por quase $23 \%$ dos meninos e $27 \%$ das meninas.

Tabela 2. Idade e as medidas antropométricas dos adolescentes por gênero. Montes Claros, 2011.

\begin{tabular}{lcccc}
\hline & $\begin{array}{c}\text { Feminino }(\mathbf{N}=\mathbf{3 6 4}) \\
\text { Média } \pm \text { DP }\end{array}$ & $\begin{array}{c}\text { Masculino }(\mathbf{N}=\mathbf{1 7 1}) \\
\text { Média } \pm \mathbf{D P}\end{array}$ & Teste t & Valor de P \\
\hline Idade (anos) & $13,45 \pm 1,3$ & $13,40 \pm 1,2$ & 0,384 & 0,701 \\
Peso $(\mathrm{kg})$ & $49,11 \pm 11,0$ & $47,8 \pm 12,3$ & 1,191 & 0,235 \\
Estatura $(\mathrm{cm})$ & $1,56 \pm 0,7$ & $1,58 \pm 0,1$ & $-1,922$ & 0,056 \\
IMC $\left(\mathrm{Kg} / \mathrm{m}^{2}\right)$ & $20,04 \pm 3,8$ & $18,93 \pm 3,5$ & 3,236 & $0,001^{*}$ \\
\hline
\end{tabular}

* diferença estatística significativa. 
Tabela 3. Distribuição do perfil antropométrico dos adolescentes, estratificado por gênero. Montes Claros, MG, 2011.

\begin{tabular}{|c|c|c|c|c|c|}
\hline \multirow[t]{2}{*}{ Estado nutricional } & \multicolumn{2}{|c|}{ Feminino $(n=364)$} & \multicolumn{2}{|c|}{ Masculino $(n=171)$} & \multirow{2}{*}{$\begin{array}{l}\text { Valor } \\
\text { de P }\end{array}$} \\
\hline & $\mathrm{n}$ & $\%$ & $\mathrm{n}$ & $\%$ & \\
\hline Baixo Peso & 14 & 3,8 & 19 & 11,1 & $0,001^{*}$ \\
\hline Eutrófico & 279 & 76,7 & 124 & 72,5 & 0,301 \\
\hline Sobrepeso & 45 & 12,4 & 20 & 11,7 & 0,826 \\
\hline Obesidade & 26 & 7,1 & 8 & 4,7 & 0,276 \\
\hline
\end{tabular}

" diferença estatística significativa.

Tabela 4. Caracterização do consumo alimentar (média $\pm \mathrm{dp}$ ) dos adolescentes e comparação entre os gêneros. Montes Claros, MG, 2011.

\begin{tabular}{lccrc}
\hline \multicolumn{1}{c}{ Nutrientes } & Feminino & Masculino & Teste t & Valor de P \\
\hline Energia (Kcal) & $3116,0 \pm 1678,5$ & $3054,3 \pm 1570,3$ & 0,405 & 0,686 \\
Carboidratos (g) & $456,9 \pm 252,8$ & $461,5 \pm 233,3$ & $-0,199$ & 0,843 \\
Proteína (g) & $100,6 \pm 59,1$ & $102,6 \pm 59,2$ & $-0,364$ & 0,716 \\
Lipídio (g) & $100,4 \pm 64,7$ & $102,9 \pm 70,8$ & $-0,391$ & 0,696 \\
Cálcio (mg) & $793,2 \pm 530,5$ & $966,3 \pm 1308,9$ & $-2,174$ & $0,030^{*}$ \\
Ferro (mg) & $15,0 \pm 8,6$ & $15,1 \pm 8,4$ & $-0,133$ & 0,895 \\
Vitamina C (mg) & $302,7 \pm 303,1$ & $290,3 \pm 261,0$ & 0,460 & 0,646 \\
Vitamina A (mcg) & $274,2 \pm 203,3$ & $297,9 \pm 233,2$ & $-1,197$ & 0,232 \\
Zinco (mg) & $16,0 \pm 10,1$ & $15,7 \pm 9,2$ & 0,316 & 0,752 \\
Fibras (g) & $38,2 \pm 19,6$ & $37,8 \pm 19,4$ & 0,192 & 0,848 \\
Na (mg) & $3688,4 \pm 2949,2$ & $3623,1 \pm 2727,1$ & 0,245 & 0,807 \\
AGSA (\%) & $8,4 \pm 2,4$ & $8,1 \pm 2,2$ & 1,216 & 0,224 \\
AGPO (\%) & $1,5 \pm 0,9$ & $1,5 \pm 0,6$ & 0,266 & 0,790 \\
Colesterol (mg) & $354,1 \pm 249,6$ & $353,4 \pm 251,1$ & 0,031 & 0,976 \\
\hline
\end{tabular}

* $\mathrm{p}<0,05 ; \mathrm{AGSA}=$ Ácido Graxo Saturado; AGPO= Ácido Graxo Poliinsaturado

Tabela 5. Distribuição percentual do consumo alimentar dos adolescentes de ambos os gêneros segundo os valores de adequação. Montes Claros, MG, 2011.

\begin{tabular}{|c|c|c|c|c|c|c|}
\hline \multirow[b]{2}{*}{ Nutrientes } & \multicolumn{3}{|c|}{ Feminino } & \multicolumn{3}{|c|}{ Masculino } \\
\hline & Insuficiente & Adequado & Elevado & Insuficiente & Adequado & Elevado \\
\hline Carboidratos (g) & 1,7 & 79,1 & 19,2 & 3,5 & 73,1 & 23,4 \\
\hline Proteína (g) & 11,5 & 88,5 & - & 18,7 & 81,3 & - \\
\hline Lipídio (g) & 29,7 & 61,2 & 9,1 & 32,2 & 55,0 & 12,8 \\
\hline Cálcio (mg) & 82,9 & 17,1 & - & 78,4 & 21,6 & - \\
\hline Ferro (mg) & 11,0 & 89,0 & - & 11,7 & 88,3 & - \\
\hline Vit. C (mg) & 3,6 & 96,4 & - & 8,2 & 91,8 & - \\
\hline Vit. A (mcg) & 81,6 & 18,4 & - & 78,9 & 21,1 & - \\
\hline Zinco (mg) & 21,4 & 78,6 & - & 17,5 & 82,5 & - \\
\hline Fibras (g) & 34,4 & 65,5 & - & 36,3 & 63,7 & - \\
\hline Sódio (mg) & - & 73,1 & 26,9 & - & 77,2 & 22,8 \\
\hline AGSA (\%) & - & 80,5 & 19,5 & - & 81,2 & 18,1 \\
\hline AGPO (\%) & 100 & - & - & 100 & - & - \\
\hline Colesterol (mg) & - & 51,6 & 48,4 & - & 51,5 & 48,5 \\
\hline
\end{tabular}

* $\mathrm{p}<0,05 ; \mathrm{AGSA}=$ Ácido Graxo Saturado; AGPO= Ácido Graxo Poliinsaturado 


\section{Discussão}

Dada a variação regional em estimativas anteriores de obesidade na juventude brasileira, é essencial acompanhar as tendências da obesidade em populações alvo. Nesse sentido, o presente estudo reafirma, para uma população de adolescentes de baixa renda de Montes Claros, a tendência ao excesso de peso corporal. Esse resultado deve ser avaliado no contexto do estudo, sem a pretensão de extrapolá-lo para outras comunidades apesar da amostra ser representativa e com poder estatístico para as análises empregadas. Resultados semelhantes são reportados em outros trabalhos, refletindo o fenômeno de transição nutricional pelo qual o Brasil vem passando e que se caracteriza pelo declínio da desnutrição e o aumento do excesso de peso, inclusive entre os adolescentes ${ }^{3,13,14}$. Segundo dados da Pesquisa de Orçamentos Familiares (POF) realizada em 20082009, o aumento de peso em adolescentes de 10 a 19 anos foi contínuo nos últimos 34 anos $^{15}$.

A apresentação dos dados por gênero visa atender às possíveis diferenças decorrentes do desenvolvimento puberal e do nível de atividade física que possam refletir em mudanças na composição corporal no período da adolescência ${ }^{16}$.

Os gêneros podem ter comportamento diferente em relação ao estado nutricional. No presente estudo, proporcionalmente, as meninas apresentaram maiores valores de Índice de Massa Corporal (IMC) e menores tendências ao baixo peso.

Outros estudos também reportam importantes diferenças quanto às prevalências de sobrepeso e obesidade entre os gêneros, mas as justificativas para estas ocorrências não são bem definidas ${ }^{17-20}$. A maior prevalência de sobrepeso e obesidade entre as meninas pode estar relacionada à presença de hormônios sexuais em idades próximas à puberdade e ao aumento dos estoques de gordura corporal ${ }^{21}$. Além disso, as diferenças quanto à de prática de atividade física também podem contribuir para essas variações ${ }^{18}$.

Embora vários estudos brasileiros e internacionais mostrem que as meninas são mais susceptíveis à obesidade que os meninos, esse dado não é consenso. Há relatos que a obesidade seja inclusive mais perceptível no gênero masculino, cujo índice no Brasil passou de 3,7\% para 21,7\% entre 2008 e 2009, o que representa um acréscimo na ordem de seis vezes ${ }^{15}$. Seria, portanto, equivocado generalizar esses dados para explicar ocorrências isoladas de obesidade, específicas a comunidades com características próprias.
A partir da detecção das tendências de obesidade, a adoção da medida correta de uma dieta é uma tarefa complexa e desafiadora, especialmente tratando-se de crianças e adolescentes. Nesse sentido, ferramentas como Questionários de Frequência Alimentar (QFA) são de grande utilidade quando adotados em estudos epidemiológicos, pois permitem o conhecimento do consumo alimentar habitual de uma população e possibilitam a classificação dos indivíduos de acordo com a ingestão de alimentos. Por isso, esse instrumento é especialmente aplicável em estudos que relacionem a dieta a efeitos na saúde em longo pra$\mathrm{zO}^{22}$. No presente estudo, optou-se por aplicar esse instrumento para caracterizar a população estudada, o que é importante visto a escassez de estudos brasileiros que validem o QFA especialmente para aqueles envolvendo adolescentes.

$\mathrm{Na}$ análise do consumo alimentar dos adolescentes, os valores elevados de desvio-padrão indicam a grande variação de consumo entre os integrantes da amostra. Resultado similar foi observado por outros autores na análise do consumo alimentar em adolescentes ${ }^{14,23}$.

Os adolescentes amostrados tinham um alto consumo energético, que superava $3000 \mathrm{kcal}$ por dia. Outros estudos realizados com questionário de avaliação mostraram que estudantes de escolas públicas no estado de São Paulo tinham um consumo energético ainda mais alto, de $3716 \mathrm{Kcal}$ para o gênero masculino e 3585 Kcal para o gênero feminino $^{24}$. O consumo substancial de energia entre estudantes brasileiros de diferentes regiões é um fator preocupante considerando que a principal fisiopatologia da obesidade é o balanço positivo no teor energético da dieta. Esse assunto, portanto, merece investigação controlada mais profunda.

$\mathrm{O}$ consumo de macronutrientes não ficou acima dos limites máximos recomendados na maioria dos adolescentes. Ainda assim, é importante verificar a tendência para os desvios apresentados no sentido de prevenir agravos nutricionais. No caso específico de carboidratos é comum que sejam consumidos em excessivo por adolescentes visto os altos níveis de açúcares na dieta, caracterizando um padrão alimentar inadequado e de risco à saúde. Um agravante nesse cenário é o aumento do consumo de bebidas à base de açúcar entre crianças e adolescentes, as quais, além de substituir opções mais saudáveis como o leite, promovem ganho de peso, diabetes, cáries dentárias e outros ${ }^{25}$.

Os adolescentes entrevistados não apresentaram alto consumo de proteínas e lipídeos. Esses nutrientes devem ser oferecidos em quanti- 
dades adequadas durante o crescimento uma vez que sua deficiência pode comprometer o desenvolvimento na puberdade. O consumo ideal de gorduras em relação aos outros macronutrientes, particularmente os carboidratos, é uma das principais questões discutidas no campo da nutrição. Diminuindo-se o conteúdo de algum nutriente específico, altera-se toda a composição da dieta, e dietas hipolipídicas em especial são frequentemente hiperglicídicas ${ }^{26}$. Nesse sentido, seria interessante garantir, na população estudada, que uma maior proporção de jovens atingisse os níveis adequados de proteínas e lipídeos.

O consumo de cálcio foi insuficiente para maioria dos meninos e meninas, que é também reportado em outros estudos com adolescentes brasileiros ${ }^{14,23}$. Este é, de fato, um problema crítico entre os adolescentes e deve estar relacionado ao baixo consumo de alimentos fontes de cálcio, como leite e derivados e vegetais verde-escuros (couve manteiga, espinafre e brócolis). O cálcio proveniente da dieta é a única fonte disponível para o organismo humano, sendo importante garantir uma ingestão mínima do mineral para o completo crescimento e maturação dos ossos, especialmente na adolescência, quando ocorre o pico de aquisição de massa óssea ${ }^{27}$.

Também foi insuficiente o consumo de vitamina A pelos adolescentes. Estudos recentes descrevem que muitos micronutrientes como a vitamina A e o cálcio estão envolvidos em processos metabólicos e endócrinos relacionados ao desenvolvimento e controle do excesso de peso. Por este motivo, a ingestão dietética adequada tornase fundamental como fator de proteção para ocorrência de obesidade ${ }^{7}$.

Outro elemento detectado no presente estudo e que pode contribuir para a promoção do sobrepeso e obesidade é o baixo consumo de fibra alimentar, que em geral é acompanhado de hábitos alimentares inadequados. As preferências alimentares dos adolescentes tendem ao reduzido consumo de frutas e hortaliças e consumo excessivo de gorduras e de doces, levando ao consumo de alimentos com quantidade elevada de carboidratos refinados e lipídeos e com baixo teor de fibra alimentar ${ }^{24}$.

Deve-se, porém, atentar ao tipo de gordura consumido. Todos os participantes ingeriam ácidos graxos poliinsaturados em quantidades insuficientes, porém uma grande proporção consumia colesterol acima das quantidades recomendadas. Também, o consumo de sódio pelos ado- lescentes ficou acima do recomendado em uma fração representativa (cerca de 26\% dos adolescentes). O baixo consumo de ácidos graxos poliinsaturados associado ao alto consumo de colesterol e sódio contribuem para a baixa qualidade da alimentação dos adolescentes, caracterizando uma dieta aterogênica que, em longo prazo, pode provocar doenças cardiovasculares ${ }^{28}$.

\section{Conclusão}

A população estudada, representativa de adolescentes matriculados na rede pública de ensino de Montes Claros e predominantemente pertencentes à classe $\mathrm{C}$, mostrou alta prevalência de excesso de peso corporal, principalmente entre as meninas. Entre os hábitos alimentares mais inadequados identificados destaca-se o alto consumo de carboidratos, ácido graxo saturado e sódio e consumo insuficiente de cálcio, vitamina A, fibras e ácido graxo poli-insaturado.

\section{Colaboradores}

L Pinho participou da concepção, planejamento, da execução da pesquisa, da análise e interpretação dos dados e da elaboração e revisão do artigo. EF Flávio e SHS Santos colaboraram na concepção do projeto e com a revisão crítica do artigo. ACC Botelho e AP Caldeira participaram da concepção, do planejamento e da orientação da pesquisa, da análise e interpretação dos dados e da elaboração e revisão do artigo.

\section{Agradecimentos}

À Coordenação de Aperfeiçoamento de Pessoal de Nível Superior (CAPES) pelo apoio financeiro. À Prefeitura Municipal de Montes Claros, MG pelo apoio logístico na realização deste estudo. 


\section{Referências}

1. Lobstein T, Baur L, Uauy R. Obesity in children and young people: a crisis in public health. Obes Rev 2004; 5(Supl. 1):S4-85.

2. Ogden CL, Carroll MD, Kit BK, Flegal KM. Prevalence of obesity and trends in body mass index among US children and adolescents, 1999-2010. JAMA 2012; 307(5):483-490.

3. Duncan S, Duncan EK, Fernandes RA, Buonani C, Bastos KD, Segatto AF, Codogno JS, Gomes IC, Freitas IF. Modifiable risk factors for overweight and obesity in children and adolescents from São Paulo, Brazil. BMC Public Health 2011; 11:585.

4. Enes CC, Slater B. Obesidade na adolescência e seus principais fatores determinantes. Rev Bras Epidemiol 2010; 13(1):163-171.

5. Moreno LA, Rodriguez G, Fleta J, Bueno-Lozano M, Lazaro A, Bueno G. Trends of dietary habits in adolescents. Crit Rev Food Sci Nutr 2010; 50(2):106-112.

6. Amuna P, Zotor FB. Epidemiological and nutrition transition in developing countries: impact on human health and development. Proc Nutr Soc 2008; 67(1):82-90.

7. Aguirre-C ML, Castillo-D C, Le Roy-O C. Desafíos Emergentes en la Nutrición del Adolescente. Rev Chil Pediatr 2010; 81(6):488-497.

8. Instituto Brasileiro de Geografia e Estatística (IBGE). Pesquisa nacional de saúde do escolar. Rio de Janeiro: IBGE; 2009. [página na Internet]. [acessado 2012 set 14]. Disponível em: http://www.ibge.gov.br/ home/estatistica/populacao/pense/pense.pdf

9. de Onis M, Onyango AW, Borghi E, Siyam A, Nishida C, Siekmann J. Development of a WHO growth reference for school-aged children and adolescents. Bull World Health Organ [Internet]. 2007 [acessado 2012 set 17]; 85:660-667. Disponível em: http:// www.who.int/bulletin/volumes/85/9/07-043497/en/ index.html

10. Slater B, Fisberg RM, Philippi ST, Latorre MRDO. Validation of a semi-quantitative adolescents food frequency questionnaire applied at a public school in São Paulo, Brazil. Eur J Clin Nutr 2003; 57(5):629-635.

11. Otten JJ, Hellwig JP, Meyers LD. Dietary reference intakes: the essential guide to nutrient requirements. Washington: National Academy Press; 2006.

12. Gidding SS, Dennison BA, Birch LL, Daniels SR, Gilman MW, Lichtenstein AH, Rattay KT, Steinberger J, Stettler N, Horn LV. Dietary recommendations for children and adolescents: a guide for practitioners. Pediatrics 2006; 117(2):544-559.

3. Cintra IP, Passos MAZ, Fisberg M, Machado HC. Evolução em duas séries históricas do índice de massa corporal em adolescentes. J Pediatr 2007; 83(2):157-162.

14. Bertin RLB, Karkle ENL, Ulbrich AZ, Neto AS, Bozza R, Araujo IQ, Campos W. Estado nutricional e consumo alimentar de adolescentes da rede pública de ensino da cidade de São Mateus do Sul, Paraná, Brasil. Rev Bras Saude Matern Infant 2008; 8(4):435-443.

15. Instituto Brasileiro de Geografia e Estatística (IBGE). Pesquisa de orçamentos familiares 2008-2009. Rio de Janeiro: IBGE; 2010. [página na Internet]. [acessado 2012 set 14]. Disponível em: http://www.ibge. gov.br/home/estatistica/populacao/condicaodevida/ pof/2008_2009/POFpublicacao.pdf
16. Romero A, Slater B, Florindo AA, Latorre MRDO, Cezar C, Silva MV. Determinantes do índice de massa corporal em adolescentes de escolas públicas de Piracicaba, São Paulo. Cien Saude Colet 2010; 15(1):141-149.

17. Guedes DP, Neto JTM, Almeida MJ, Silva AJRM. Impacto de fatores sociodemográficos e comportamentais na prevalência de sobrepeso e obesidade de escolares. Rev Bras Cineantropom Desempenho Hum 2010; 12(4):221-231.

18. Tassitano RM, Barros MVG, Tenório MCM, Bezerra J, Hallal PC. Prevalência e fatores associados ao sobrepeso e à obesidade em adolescentes, estudantes de escolas de Ensino Médio de Pernambuco, Brasil. Cad Saude Publica 2012; 25(12):2639-2652.

19. Gupta N, Goel K, Shah P, Misra A. Childhood obesity in developing countries: epidemiology, determinants, and prevention. Endocr Rev 2012; 33(1):48-70.

20. Adesuwa FA, Oliemen P, Ifeoma A, Nwadiuto AA. Weight status of adolescents in secondary schools in port Harcourt using Body Mass Index (BMI). Ital J Pediatr 2012, 38:1.

21. Biro FM, Khoury P, Morrison JA. Influence of obesity on timing of puberty. Int J Androl 2006; 29(1):272277.

22. Magarey A, Watson J, Golley RK, Burrows T, Sutherland R, Mcnaughton SA, Denney-Wilson E, Campbell K, Collins C. Assessing dietary intake in children and adolescents: Considerations and recommendations for obesity research. Int J Pediatr Obes 2011; 6(1):2-11.

23. Chiarelli G, Ulbrich AZ, Bertin RL. Composição corporal e consumo alimentar de adolescentes da rede pública de ensino de Blumenau (Brasil). Rev Bras Cineantropom Desempenho Hum 2011; 13(4): 265-227.

24. Toral N, Slater B, Silva MV. Consumo alimentar e excesso de peso de adolescentes de Piracicaba, São Paulo. Rev Nutr 2007; 20(5):449-459.

25. Sales-Peres SHC, Goya S, Sant'Anna RMF, Silva HM, Sales-Peres AC, Silva RPR, Lauris JRP, Bastos JRM. Prevalência de sobrepeso e obesidade e fatores associados em adolescentes na região centro-oeste do estado de São Paulo (SP, Brasil). Cien Saude Colet 2010; 15(Supl. 2):3175-3184.

26. Ferreira AV, Mario EG, Porto LC, Andrade SP, Botion LM. High-carbohydrate diet selectively induces tumor necrosis factor-á production in mice liver. Inflammation 2011; 34(2):139-145.

27. Leão ALM, Santos LC. Consumo de micronutrientes e excesso de peso: existe relação? Rev Bras Epidemiol 2012; 15(1):85-95.

28. Dishchekenian VRM, Escrivão MAMS, Palma D, Ancona-Lopez F, Araújo EAC, Taddei JAAC. Padrões alimentares de adolescentes obesos e diferentes repercussões metabólicas. Rev Nutr 2011; 24(1):17-29.

Artigo apresentado em 28/08/2012

Aprovado em 29/09/2012

Versão final apresentada em 26/10/2012 